\title{
A Voronoi Based Coverage Enhancement for Wireless Sensor Networks
}

\author{
Blessington J., Komban B., Lakshmiprabha M., Monisha A., Subramanian K.
}

\begin{abstract}
Wireless sensor network which competes with the modern technologies also paves the way for research and commercial development. Mobile and static sensors form a network that balances sensor coverage and the cost of the sensor. For this a thorough study of the coverage area and mobility of mobile sensor node is necessary. Coverage in wireless networks invoke the observation of physical distance enclosed by the sensors. Voronoi diagrams are used to find out the coverage holes and design movement - assisted sensor deployment protocols, VEC that works on the principle of moving sensors whereas Voronoi based works on the basis of load balancing. The algorithms gain sensor energy stabilization and small effect of deployment energy utilization. Its effectiveness is examined in terms of coverage, uniformity, time and distance. Key words Sensor deployment,
\end{abstract}

Keywords: Sensor deployment, mobile sensors, voronoi diagram, energy consumption

\section{INTRODUCTION}

A wireless sensor network is a compilation of nodes which comprises of autonomous sensing devices which are spatially arranged and used to record physical or environs parameters (temperature, sound, pressure etc.)

Each node consists of:

- Processing capability (one or more microcontrollers, CPU's or DSP chip's)

- Multiple types of memory (program, data and flash memories),

- RF transceiver(with a single Omnidirectional antenna),

- Power source(batteries),

- Sensors and actuators.

\section{ADVANTAGES OF WSN}

- Detecting intrusion

- Biotelemetry

- Managing traffic loads

- Monitoring health and human activity patterns.

\section{ISSUES IN WSN}

- Power resource control to extend the lifetime of the network.

Revised Manuscript Received on February 05, 2020.

* Correspondence Author

Blessington J.*, Department of Electronics and Communication Engineering, National Engineering College, Tamil Nadu, India.

Komban .K, Department of Electronics and Communication Engineering, National Engineering College, Tamil Nadu, India.

Lakshmiprabha .M, Department of Electronics and Communication Engineering, National Engineering College, Tamil Nadu, India.

Monisha .A, Department of Electronics and Communication Engineering, National Engineering College, Tamil Nadu, India.

Subramanian .K, Assistant Professor, National Engineering College, Kovilpatti, Tamilnadu, India.

(C) The Authors. Published by Blue Eyes Intelligence Engineering and Sciences Publication (BEIESP). This is an open access article under the CC BY-NC-ND license (http://creativecommons.org/licenses/by-nc-nd/4.0/)
- Coverage and Connectivity.

Coverage holes area could also be created after random usage of sensors within the monitoring area or limited power source of sensors were consumed in wireless sensor network (WSN) hence, some research works propose way to recover the coverage holes area and maintaining connectivity by improving the sensing range or movement assisted sensor deployment. Finally it showed some algorithms to reinforce coverage, and connectivity with mobile nodes without human deployed.

In an environment here the facility resource is batteries, which will consider wireless sensors are situated in a neighborhood of interest that has to be rexamined whether during a random or known fashion. The sensors formulate themselves during a radio network employing a routing algorithm to examine the world for whatever limitations it was made to visually examine, and broadcast the information to a central node, sometimes called a base station, or sink node, that collects the information from all of the sensors. This node could also be an equivalent because the other nodes, or due to increased conditions, could also be a more powerful node with elevated power. The greatest leverage of wireless sensors is that they will be arranged in an environment for enhanced periods of time, continually focusing the environment, without the necessity for human involvement or funtions. This, however, initializes the facility source as the limiting element of the sensor.

For the distribution and attaiment, the wireless sensor network coverage is the critical issue pointing the quality of surveillance that the network can provide. For example how well the sensors can monitor a region of interest and how efficacy a sensor network can detect interloper. In order to enhance design and use sensor and use sensor networks in different application scenarios it is essential to explain how a coverage ${ }^{[10]}$ of a sensor network depend on various network features.

\section{RELATED WORKS}

There are several factors one should consider while sensor deployment, the most important thing is coverage and energy consumption. Through mere deployment in any target area, coverage can be barely achieved ${ }^{[1]}$. There are several factors one should consider while deploying sensor, the most important thing is coverage and energy consumption. Through mere deployment in any targeted area, coverage can be barely achieved .coverage can also be achieved by mobile sensors but there will be a reduction in energy of the nodes while they shift their position and coverage area.

\section{Published By:}

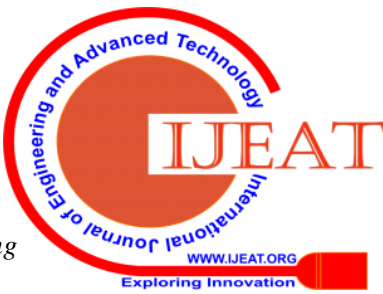


In mobile sensors are kept to act according to the control which is given by the static sensor and is followed by vector based approach. Its duplicate healing is one of the major problems dealt here. In order to increase the coverage the number of rounds is increased which leads to this problem and it was found that the duplicate healing is decreased. The static and mobile sensor mixture requires a keen watch on the cost of the sensors with the limitations prevailing.

Bidding protocols are designed for deployment of mobile sensors $^{[2]}$ in addition with static sensor nodes to ensure adequate coverage. The static sensor node decides the movement of each mobile sensor nodes by providing the estimated whole size in coverage. To acquire energy consumption in addition with coverage bidding protocols are described. To cover a hole the mobile sensors are moved in each and every round.

To increase the energy consumption the sensors are moved in a zig-zag manner. A proxy based bidding protocol has been designed to avoid that, where the mobile sensor moves only after their final location is resolved. In a proxy based bidding protocol the weakness of the bidding protocol in movement and energy consumption is shot through by virtual movement which provides the same coverage. To diminish the average moving distance and to avoid multiple healing coverage hole exchange is introduced. To adopt the effective one both distance based and cost based approach are considered in the movement of the sensor.

The largest bid is accepted by the mobile sensors and it gives the highest priority to be healed. In ample trade-off between sensor cost and coverage are treated as the primary constraints.

Under various conditions and specifications three different the round number when all sensors stop, E - the efficacy of moving, which is the rate of actual moving distance to the distance between the initial position and the final position, $\mathrm{M}$ - the average number of movements of sensors. C - the coverage and $\mathrm{D}$ - the moving distance are compared for three different algorithms - Vector based algorithm, Voronoi Based algorithm and Min-Max algorithm, all algorithms are effective while considering coverage, when it comes to moving distance and average number of movements voronoi based algorithm will be the finest one. The critical factor for WNS is considered as coverage. In the first instance reduced number of nodes is placed and the region is being divided into irregular polygons through voronoi approach. Each node in a particular voronoi region tries to cover the whole polygon fails patently ${ }^{[9]}$. While deploying more sensors expecting more coverage the number of nodes in each polygon is increased. The performance characteristics for different number of nodes are obtained in each polygon and it gives the optimum number of sensors to be deployed for extreme coverage.

\section{BACKGROUND DETAILS}

\section{Deployment Protocols:}

After the deployment of both static and mobile sensors to improve the coverage we implement two different protocols to distribute sensors across the targeted areas ${ }^{[4]}$

i) VOR -functions just in the opposite way to VEC i.e. attracts the sensors to the sparsely populated area. ii) VEC approaches are being compared for their performance. $\mathrm{R}$ -

densely populated sensors are scattered away, it is a proactive algorithm.

\section{Voronoi based algorithm:}

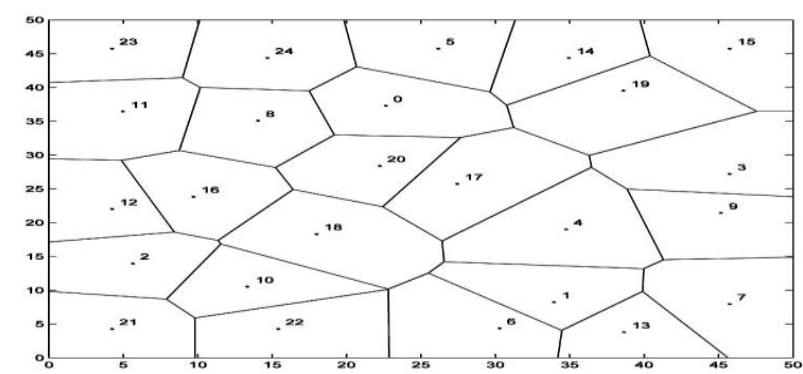

(a)

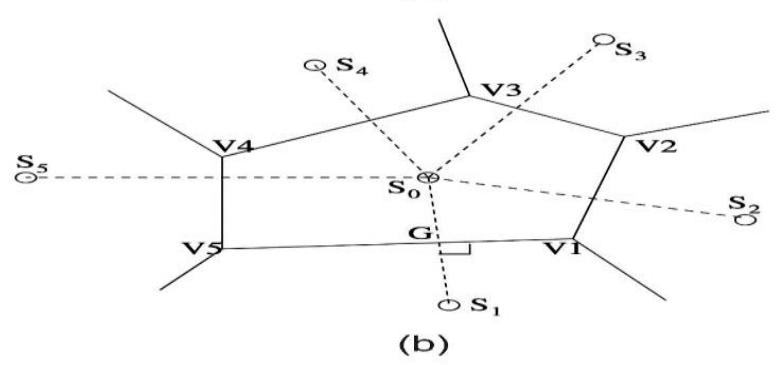

Fig. 1.(a) Voronoi diagram (b) Voronoi polygon

The target area is split into irregular polygons known as VORONOI 5 regions. Each point in a given polygon is more proximate to the node in this polygon than to any other node. We define the Voronoi polygon of $\mathrm{O}$ as $\mathrm{G}(\mathrm{O})=$ $(\mathrm{V}(\mathrm{O}), \mathrm{E}(\mathrm{O}))$, where $\mathrm{V}(\mathrm{O})$ is the set of Voronoi vertices of $\mathrm{O}$, and $E(O)$ is the set of Voronoi edges. As shown in Fig. 1(b), $\mathrm{V}(\mathrm{O})=\{\mathrm{V} 1, \mathrm{~V} 2, \mathrm{~V} 3, \mathrm{~V} 4, \mathrm{~V} 5\}, \mathrm{E}(\mathrm{O})=\{\mathrm{V} 1 \mathrm{~V} 2, \mathrm{~V} 2 \mathrm{~V} 3$, V3V4, V4V5, V5V1 $\}, \mathrm{N}(\mathrm{O})=\{\mathrm{A}, \mathrm{B}, \mathrm{C}, \mathrm{D}, \mathrm{E}\}$, where $\mathrm{N}(\mathrm{O})$ denotes the set of Voronoi neighbors of $\mathrm{O}$. The Voronoi edges of $\mathrm{O}$ are the vertical bisectors of the line passing $\mathrm{O}$ and its Voronoi neighbors, e.g., V5V1 is OA's bisector. All the points inside $\mathrm{G}(\mathrm{O})$ are closer to $\mathrm{O}$ than to any other nodes. In voronoi based algorithm ${ }^{[5]}$ when a hole is detected by a sensor it will move towards its extreme voronoi vertex.

\section{Vector-Based Algorithm}

VEC improves the coverage of target area by moving the sensors by applying the attributes of electromagnetic particles. The expected action of electromagnetic particles is to repel each other when 2 such particles are very much close to each other. Consider the average distance between the two sensors si and $s j$, be $d\left({ }_{s i}, s_{j}\right)$. $d_{a v e}$ be the average distance between 2 sensors when they are evenly dispersed in the destination area. The force pushes the sensors to move $\left(d_{a v e}-d\left(s_{i}, S_{j}\right)\right) / 2$ far each other. ${ }^{[3]}$ There may scenarios where one of the sensors provide full coverage for its own polygon then it should not move. In such cases the other sensor will be moved away to a distance of $\mathrm{d}_{\mathrm{ave}}-\mathrm{d}\left(\mathrm{si}, \mathrm{s}_{\mathrm{j}}\right)$. Before moving, the sensor determines if its local coverage increases when it shift to the new positionu. If there is no such improvement the sensor should not move to the destination location. In addition to the force exerted by the sensors, 
there will be forces which will be exerted by the boundaries which will move the sensors inwards to the polygon. This force will move the sensor to a distance of $\mathrm{d}_{\mathrm{ave}} / 2-\mathrm{db}\left(\mathrm{s}_{\mathrm{i}}\right)$, where $d b\left(s_{i}\right)$ is the distance of $s_{i}$ to the frontier inward, $d_{a v e} / 2$ is the distance from the frontier to the sensors nearest to it when they are evenly dispersed.

\section{PROPOSED SYSTEM}

\section{ENERGY EFFICIENT MECHANISM:}

For a movement of each unit a sensor is set to make use of $0.003 \%$ of its energy and all the mobile sensors are initially assumed to have ideally $100 \%$ of energy level. For a sensor to move in vector based methodology in energy efficient algorithm the criteria is that it should have energy level more than $60 \%$ if not the next nearby sensor node with higher energy level is moved to cover the hole

\section{MOVEMENT OF NODES BASED ON PENALTY VALUE}

The sensor nodes are deployed inconstantly in the target area and moved based on vector based algorithm. The figure shows how the sensor nodes are penalized if they are nearby the region under the coverage of one sensor will be as same as that of another sensor if the sensor nodes are placed as if their distance is very low. So this project is designed in such a way to lessen the number of sensors penalized. So to avoid the sensor getting penalized in vector based algorithm, the minimum distance in which the algorithms operates is set as $2 r$, where $r$ is the radius of the coverage circle of the mobile sensor.

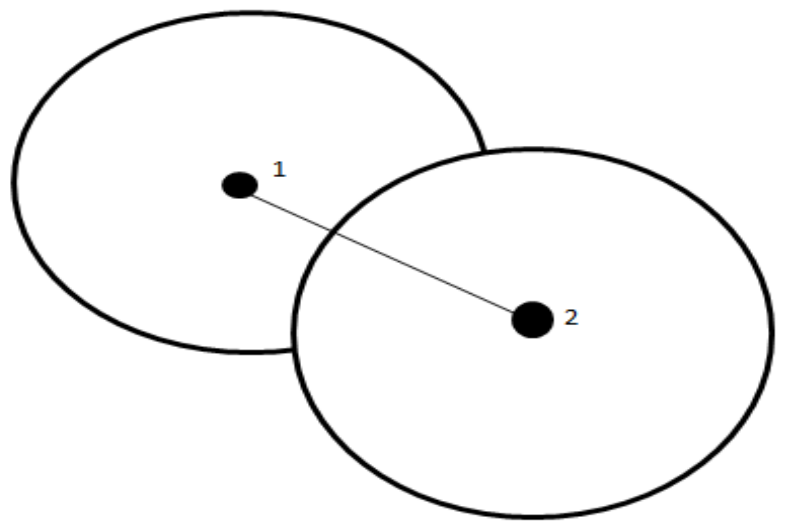

Fig. 2.Penalizing of coverage of mobile sensors

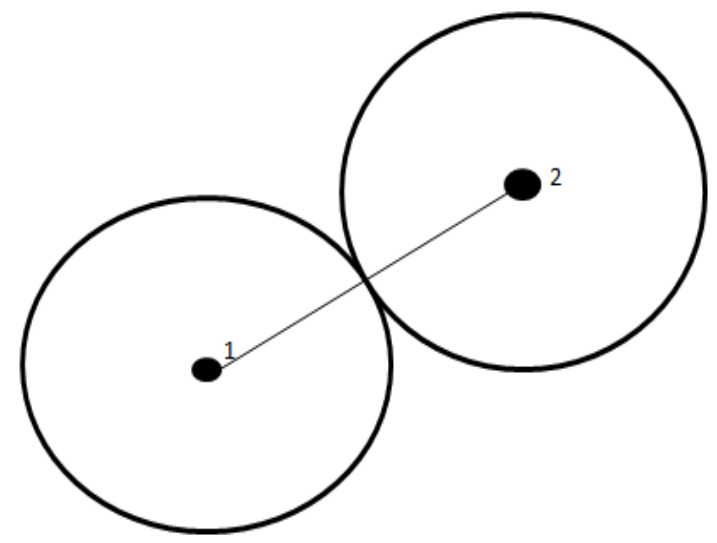

Fig. 3.Healing penalty

\section{ENERGY EFFICIENT ALGORITHM:}

The crucial parameter in designing any wireless sensor networks is energy consumption. For a long duration the sensor node deployed should withstand. In addition the distance moved by the mobile nodes in order to provide adequate coverage should also be reduced as it increases energy consumption ${ }^{[4]}$.There should be optimum distance between the nodes in any wireless sensor networks so that the coverage provided by one sensor node is not penalized by the other. Load balancing factor which is the ratio between the numbers of nodes penalized at initial deployment to that of the final movement is introduced in our project. How the penalty of the coverage can be reduced is determined by this factor.

When compared to the existing system energy efficient algorithm reduces the average energy consumption around 4 to $5 \%$.one of the inevitable advantage is reducing energy consumption up to $5 \%$ for each mobile node so that the mobile nodes withstand for longer duration. Optimum coverage can also be provided. By this algorithm we can provide the coverage that is provided by the existing system in addition with efficient energy consumption. The movement of mobile nodes should be optimal and a crucial parameter in wireless sensor networks which consist of mobile nodes. The movement of nodes is more important to provide ideal coverage in the target area. But movement is a path way for energy utilization and so the decrement in energy level of the individual sensors.

The average moving distance is considerably reduced around 20 units compared to the existing system in this algorithm $^{[6]}$. In reducing the energy consumption a considerable factor is reducing moving distance of mobile nodes about 20 units. In order to provide optimum coverage needed in the target area in addition with reduction in moving distance and energy consumption the load balancing factor is reduced means penalty of coverage of sensor nodes is considerably reduced in this algorithm.

\section{WORK FLOW OF ENERGY EFFICIENT ALGORITHM FOR WSN's:}

The region under the target area or surveillance is identified and static nodes are inconstantly arranged in the region. The destination area is divided into voronoi polygons by means of the position of the static nodes as each polygon consists of a static node. To improve coverage provided mobile nodes are positioned irregularly in the target area. Later initial implementation here it is assumed that all the mobile sensors have $100 \%$ energy level ${ }^{[7]}$. And also it is assumed that for moving a mobile sensor of a unit it consumes energy level of $0.003 \%$.In order to remove the penalty of coverage of one sensor by its counterpart mobile nodes are moved in the basis of vector based algorithm. In the basis of vector based algorithm moving a mobile node it is mandatory to ensure that the mobile node to be moved has enough energy level to withstand. It is proposed to move the immediate nearby sensor which has enough energy level if a sensor node is to be moved is having energy level less than $60 \%$. In order to heal the holes and to avoid the penalty of coverage by considering energy consumption as the crucial parameter the entire nodes are moved.

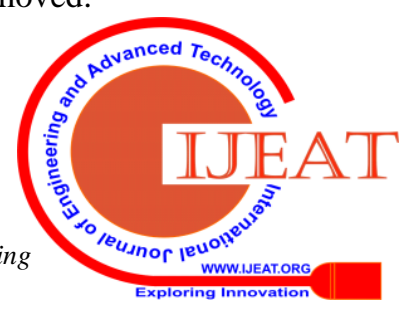




\section{V.DETERMINATION OF COVERAGE}

In the proposed model it is assumed that the coverage area of static sensor is within a circular area of radius 125 units and for mobile sensors it is within a circular area of radius 100 units.

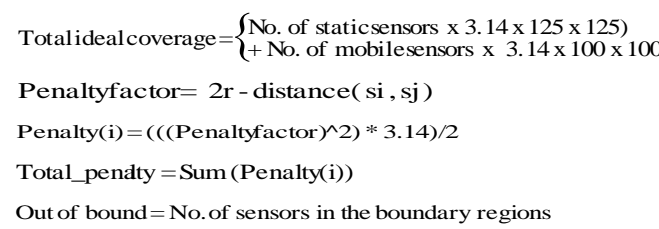

Fitness is the practical coverage that can be provided by the WSN in the target area and it is improved to a greater extent in each rounds and optimum coverage is attained in the final round.

\section{PERFORMANCE EVALUATION}

In the region under surveillance the static wireless sensor nodes have been deployed randomly.

\section{STATIC NODES WITHIN THE VORONOI POLYGON}

The Voronoi diagram is a compilations of nodes segregating the space into polygons. In a given polygon every point is nearer to the node in this polygon than to any other node. Voronoi diagram can be used to find potential coverage holes.

\section{COMMUNICATION BETWEEN NODES}

This figure shows how the communications are done between all the nodes. The factor which decides the communication between the static nodes is the distance between the individual nodes and is denoted as range. If the distance is less than the range value there will be communication between the nodes.

\section{STATIC AND MOBILE NODES ON VORONOI POLYGON:}

The green shades indicate static nodes and the Blue shades indicate mobile nodes. Each sensor indicated by a number, which is overlaid by a voronoi polygon. These polygons collectively cover the target field.

\section{MOVEMENT OF NODES USING VECTOR BASED ALGORITHM:}

The mobile nodes are moved bases on vector based algorithm indent to maximize the coverage and minimize the movements. The entire algorithm is split into number of rounds and finally the round terminates when there is no improvement in coverage in two successive rounds.

Total number of rounds: 5

Total number of iterations: 1980 for 5 rounds
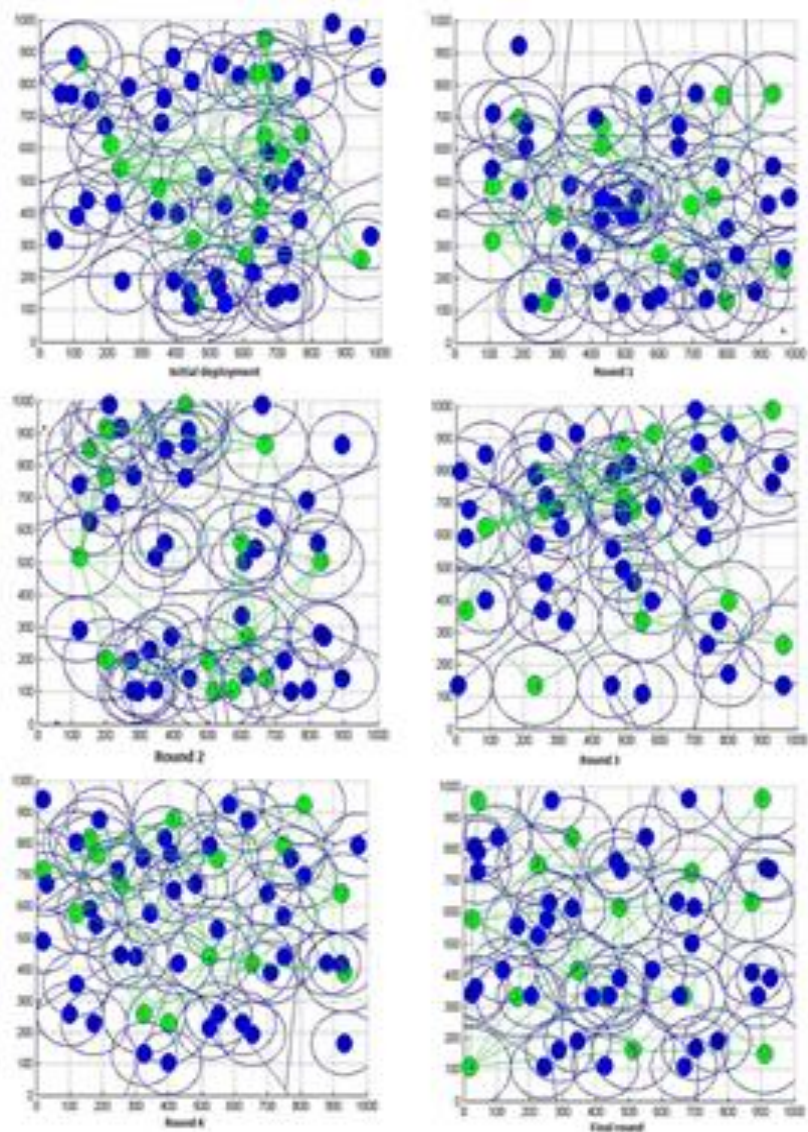

Fig. 4.Position of nodes

Figure 5.1 - Position of nodes Initial Deployment: Initial deployment gives coverage of $73 \%$.

Round 1: Round 1 gives coverage of $78 \%$.

Round 2: Round 2 gives coverage of $83 \%$.

Round 3: Round 3 gives coverage of $87 \%$.

Round 4: Round 4 gives coverage of $90 \%$.

Round 5: The maximum coverage has been attained which is $93 \%$ in round 5 no more improvement will be provided in coverage by moving the mobile nodes and thus the final destinations of the mobile nodes have been reached. i.e. They can't be moved further.

One of the important parameter in deployment issues related WSN's is energy consumption. Energy consumption gets arise from mechanical movements of the sensors. The average moving distance is used as the metric for energy consumption $^{[8]}$. The mean value of the distance moved by each mobile sensors is average moving distance. The movement of the sensors depends on the vector based algorithm and penalty criteria. The average moving distance reduction is using energy efficient protocol with number of sensors and average moving distance reduces to a considerable extent. Average moving distance gradually raises with increase in the number of sensors deployed.

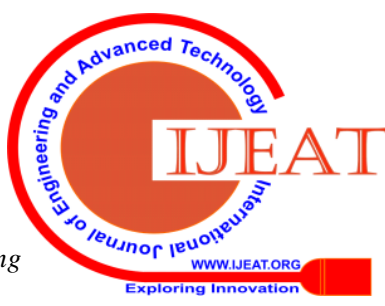




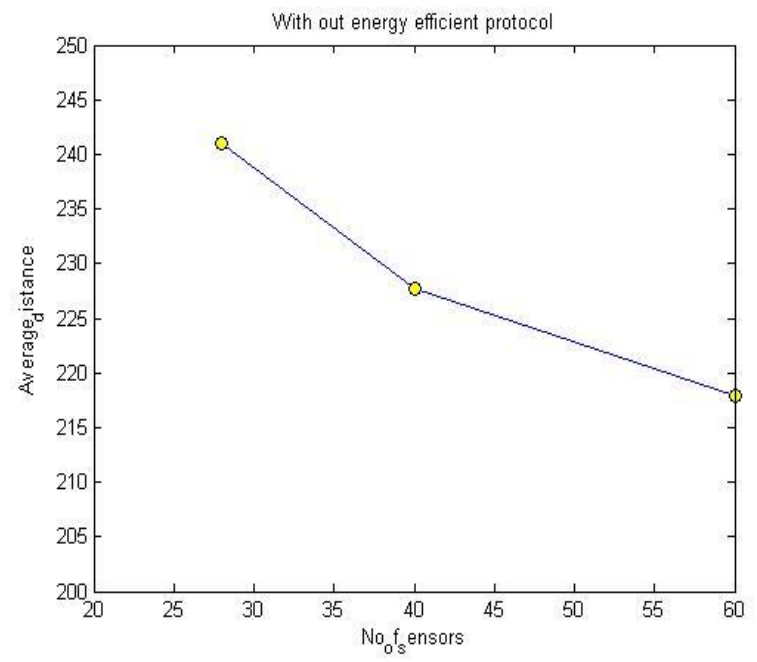

Fig. 5.Average distance Vs No. Of sensors for with energy efficient protocol

The average energy consumption is reduced to a greater extent in energy efficient algorithm. Figure shows the reduction in the energy consumption.

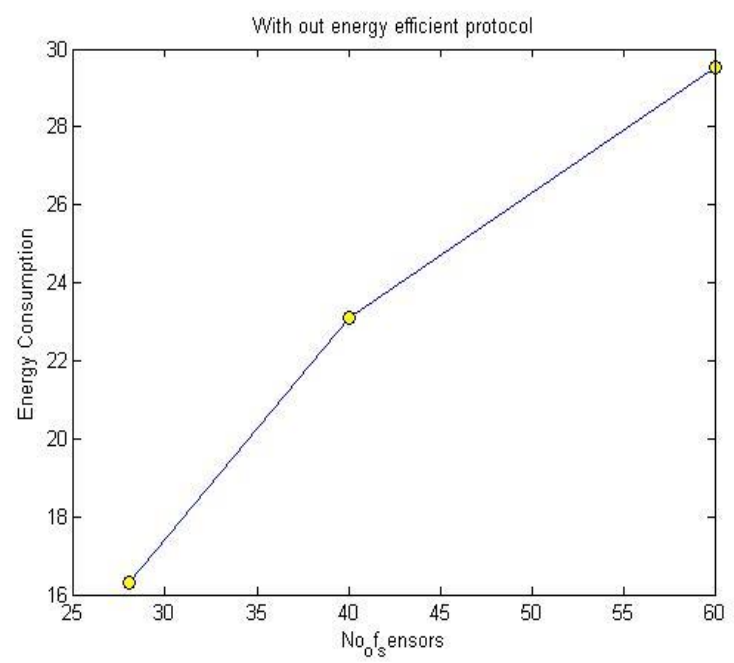

Fig. 6.Energy consumption Vs Number of sensors for without energy efficient protocol

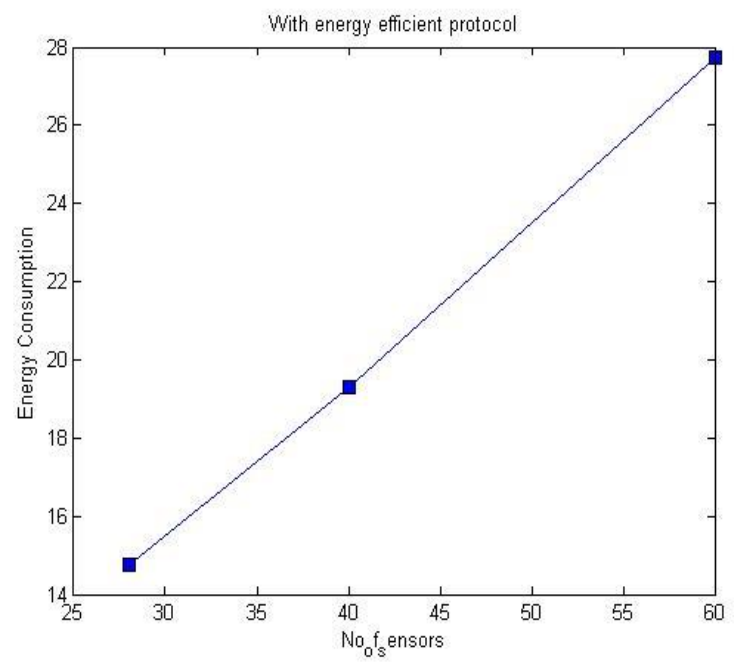

Fig. 7.Energy consumption Vs No. Of sensors for with energy efficient protocol

\section{LOAD BALANCING FACTOR:}

It is described as the rate between the number of mobile sensors penalized in the initial deployment to the same as that of the final deployment. ${ }^{[11]}$ bTo find the number of sensors penalized we use the voronoi approach which in turn used to determine the load balancing factor. Figure shows how the load balancing factor fluctuates with the number of sensors.

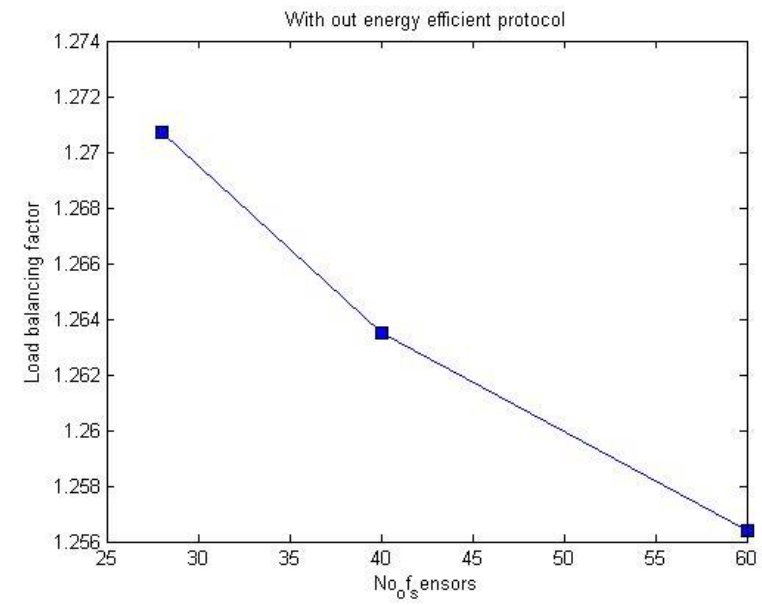

Fig. 8.Load balancing factor Vs Number of sensors for without energy efficient protocol

\section{CONCLUSION}

We have initiated energy efficiency which reduces the average moving distance and the energy consumption in addition to providing load balancing which are all crucial parameters in a wireless sensor networks. While designing protocols for wireless sensor networks coverage is the important parameter. In our project we have initiated to use vector based algorithm for movement of sensor nodes coupled with energy efficient mechanism in order to minimize energy utilization and minimizing movements. Our outcome show that our protocol is more efficient in energy consumption, coverage and reduction in movement.

\section{REFERENCES}

1. wang, g., cao, g., \& la porta, t. f. (2006). movement-assisted sensor deployment. ieee transactions on mobile computing, 5(6), 640-652.

2. wang, g., cao, g., \& la porta, t. (2004, october). proxy-based sensor deployment for mobile sensor networks. in 2004 ieee international conference on mobile ad-hoc and sensor systems (ieee cat. no. 04ex975) (pp. 493-502). ieee.

3. rahmani, n., nematy, f., rahmani, a. m., \&hosseinzadeh, m. (2011). node placement for maximum coverage based on voronoi diagram using genetic algorithm in wireless sensor networks. australian journal of basic and applied sciences, 5(12), 3221-3232.

4. banerjee, c. (2013, april). energy conservation in wireless sensor network using irregular cellular automata. in 2013 international conference on energy efficient technologies for sustainability (pp. 223-227). ieee.

5. http://sensorwebs.jpl.nasa.gov

6. http://wins.rsc.rockwell.com.

7. aurenhammer, f. (1991). voronoi diagrams - a survey of a fundamental geometric data structure. acm computing surveys (csur), 23(3), 345-405. 


\section{A Voronoi Based Coverage Enhancement for Wireless Sensor Networks}

8. meguerdichian, s., koushanfar, f., potkonjak, m., \&srivastava, m. b. (2001, april). coverage problems in wireless ad-hoc sensor networks. in proceedings ieee infocom 2001. conference on computer communications. twentieth annual joint conference of the ieee computer and communications society (cat. no. 01ch37213) (vol. 3, pp. 1380-1387). ieee.

9. zou, y., \&chakrabarty, k. (2003, march). sensor deployment and target localization based on virtual forces. in ieee infocom 2003. twenty-second annual joint conference of the ieee computer and communications societies (ieee cat. no. 03ch37428) (vol. 2, pp. 1293-1303). ieee.

10. zhao, f., guibas, l. j., \&guibas, l. (2004). wireless sensor networks: an information processing approach. morgan kaufmann.fortune, s. (1992). voronoi diagrams and delaunay triangulations. in computing in euclidean geometry (pp. 193-233).

11. fortune, s. (1992). voronoi diagrams and Delaunay triangulations.in computing in euclidean geometry (pp. 193-233).

\section{AUTHORS PROFILE}

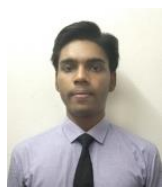

Blessington $\mathbf{J}$., is an undergraduate student in department of Electronics and communication engineering at National Engineering College, Tamil Nadu, India. His research interest includes networking.

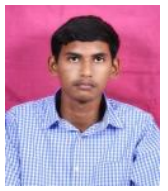

Komban .K, is an undergraduate student in department of Electronics and communication engineering at National Engineering College, Tamil Nadu, India. His research interest includes networking

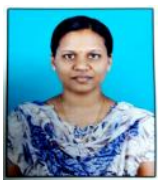

Lakshmiprabha .M, is an undergraduate student in department of Electronics and communication engineering at National Engineering College, Tamil Nadu, India. Her research interest includes networking.

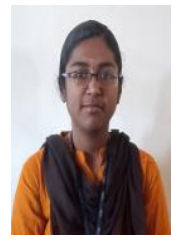

Monisha .A, is an undergraduate student in department of Electronics and communication engineering at National Engineering College, Tamil Nadu, India. Her research interest includes networking.

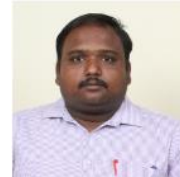

Subramanian .K, received B.E degree in Electronics and Communication Engineering from Hindusthan Engineering College and Technology (2005) and M.E degree from National Engineering College, Kovilpatti (2008). He is currently working in National Engineering College, Kovilpatti, Tamilnadu, India as Assistant Professor(SG). His research interest includes Sensor deployment and Mobile ad-hoc networks. 